\title{
Health Communication Patterns and Adherence to a Micronutrient Home Fortification Program in Rural China
}

\author{
Ruixue Ye, $\mathrm{MPH}^{1}$; Yuju Wu, $\mathrm{PhD}^{1}$; Chang Sun, $\mathrm{MPH}^{1}$; Qingzhi Wang, $\mathrm{MPH}^{1}$; \\ Yuping Mao, $\mathrm{PhD}^{2}$; Hein Raat, $\mathrm{PhD}^{3}$; Scott Rozelle, $\mathrm{PhD}^{4}$; Hannah Johnstone, $\mathrm{MA}^{4}$; \\ Huan Zhou, $\mathrm{PhD}^{1}$
}

\begin{abstract}
Objectives: Examine the association between ethnic health communication patterns and adherence to a micronutrient home fortification program in rural China among 3 distinct ethnic groups.

Design: Cross-sectional survey conducted in rural western China.

Setting: Enrolled 283 villages across 6 national poverty counties in rural western China.

Participants: A total of 1,358 caregiver-children pairs with Han, Tibetan, or Yi ethnic backgrounds.

Variables Measured: A structured questionnaire was used to collect information on caregiver demographics, program adherence, and health communication about the program.

Analysis: Logistic regression model was used to examine the associations between health communication patterns and adherence to the program.

Results: Adherence rates across all ethnic groups were low, 55.5\% (229/413) of Han, 55.0\% (186/338) of Tibetan, and 47.2\% (178/377) of Yi caregivers adhered to the program. Increased adherence was correlated with how each ethnic group received health information. Han caregivers were most influenced by mass media (odds ratio [OR], 1.87; 95\% confidence interval [CI], 1.05-3.31), Tibetan caregivers by family (OR, 4.86; 95\% CI, 1.45-16.29), and Yi caregivers by village doctors (OR, 6.63; 95\% CI, 3.46-12.73).

Conclusions and Implications: Implementing culturally sensitive health communication strategies will likely improve adherence to home fortification programs among caregivers with distinct ethnic backgrounds.

Key Words: home fortification program, adherence behavior, health communication, ethnic differences, western China (J Nutr Educ Behav. 2022;54:36-45.)
\end{abstract}

Accepted July 30, 2021. Published online October 21, 2021.

\section{INTRODUCTION}

Although the prevalence of stunting and wasting has decreased in the last decade, children aged $<5$ years in low- and middle-income countries
(LMICs) continue to suffer from micronutrient deficiencies, particularly iron-deficiency anemia. ${ }^{1,2}$ Early childhood anemia is often associated with insufficient childhood nutrition and may negatively affect health

\footnotetext{
${ }^{1}$ Department of Health Behavior and Social Medicine, West China School of Public Health and West China Fourth Hospital, Sichuan University, Chengdu, Sichuan, China

${ }^{2}$ Department of Communication Studies, College of Liberal Arts, California State University Long Beach, Long Beach, CA

${ }^{3}$ Department of Public Health, Erasmus MC, University Medical Center Rotterdam, Rotterdam, The Netherlands

${ }^{4}$ Rural Education Action Program, Freeman Spogli Institute for International Studies, Stanford University, Palo Alto, CA

Conflict of Interest Disclosure: The authors have not stated any conflicts of interest.

Address for correspondence: Huan Zhou, PhD, Department of Health Behavior and Social Medicine, West China School of Public Health and West China Fourth Hospital, Sichuan University, Chengdu, Sichuan, China, 610041; E-mail: zhouhuan@scu.edu.cn

(C) 2021 The Authors. Published by Elsevier Inc. on behalf of Society for Nutrition Education and Behavior. This is an open access article under the CC BY-NC-ND license (http:// creativecommons.org/licenses/by-nc-nd/4.0/)

https://doi.org/10.1016/j.jneb.2021.07.014
}

outcomes throughout an individual's life. ${ }^{2,3}$ In response to this nutrition crisis, the World Health Organization (WHO) recommends that countries with high rates of childhood anemia (> 20\%) distribute micronutrient powders (MNPs) to meet the nutritional needs of children. ${ }^{4}$ A number of MNP programs have since been implemented internationally, and multiple studies have found significant declines in childhood anemia because of MNP programs. ${ }^{5}$

Unfortunately, research conducted in LMICs has shown low rates of caregiver adherence to MNP programs. ${ }^{6-8}$ Although there are many possible reasons for low levels of adherence in LMICs, researchers have determined that culturally appropriate and effective health communication strategies influence MNP adherence. ${ }^{9-12}$ International 
studies have shown that for MNP programs to be successful, local characteristics, such as socioeconomic status (SES), cultural norms, and beliefs, must be taken into consideration. Studies in LMICs have found that effective communication methods within the context of one socioeconomic and cultural community may not be as effective in other contexts. For example, one study of MNP programs found that communication from village-level health teams was most effective for increasing adherence in sampled communities of rural Uganda. ${ }^{10}$ Another study conducted in rural communities in Peru indicated that different forms of communication about the benefits of MNPs from relatives and neighbors were most effective. ${ }^{11}$

In rural areas of western China, like other LMICs, research has found high rates of childhood anemia, with $>50 \%$ of infants and toddlers suffering from iron-deficiency anemia in western rural communities. ${ }^{13,14}$ To address these high anemia rates, China's government implemented its own MNP distribution program, Ying Yang Bao (YYB). The program has been implemented in rural, low SES communities in western China. These communities are composed of diverse ethnic groups, including Han (the largest ethnic group in China, comprising $>90 \%$ of the population) and a series of ethnic minority groups, including Tibetan, Yi, Bai, and Hui. ${ }^{15,16}$ This program distributes YYB packets to local township and village doctors who are in charge of delivering the packets and YYB information to local caregivers. Doctors typically distribute YYB packets and provide information about YYB to caregivers during routine infant health checks at the township health centers or village clinics. In villages, if caregivers live far from the local clinic, doctors may make home visits. ${ }^{15,17,18}$ Doctors do not follow a formal YYB communication plan and, instead, use their discretion to decide what YYB information to provide to households. In addition to doctor visits, caregivers may receive information about YYB through mass media. ${ }^{19}$ However, there are no formal communication plans to specifically accommodate different cultural contexts.
Although the YYB program receives substantial government support, adherence rates in western China remain low. Studies have found that only $36 \%$ to $50 \%$ of caregivers regularly feed their children YYB packets. ${ }^{19-23}$ Similar to other LMICs, it may be that YYB programs need to be tailored to local contexts to improve YYB adherence and reduce the overall prevalence of anemia in rural western China.

Given that the culture of an ethnic group is an important factor that shapes the values, lifestyles, and communication patterns of its people, ${ }^{24,25}$ it is likely that culturally appropriate strategies of dispersing information can improve YYB adherence in the ethnically diverse program sites in western China. However, the association between health communication patterns and YYB adherence among different ethnic groups in western China has not been studied. Therefore, the purpose of this study is to explore (1) YYB adherence rates among caregivers who are ethnically Han, Tibetan, and Yi; (2) how health information is communicated among these 3 ethnic groups; and (3) how health communication patterns are associated with YYB adherence among Han, Tibetan, and Yi caregivers.

\section{METHODS}

\section{Study Design and Participants}

Our research team conducted a crosssectional study in May 2019 in rural, multiethnic areas of Sichuan Province in western China. In this study, Han, Tibetan, and Yi communities were sampled. The multiethnic population of Sichuan Province and the unique demographic characteristics of each ethnic group make this province ideal for conducting this study.

As detailed in the Figure, a 4-step, multistage, cluster sampling method was used to select the study's sample. To choose the study sample, a computer-generated random number method was implemented at each level. First, to select the sample counties, we identified 32 YYB program counties in Sichuan Province. From these 32 counties, 2 rural Han counties, 2 rural Tibetan counties, and 2 rural Yi counties were selected. Second, we randomly selected 6 sample townships within each sampled county. Townships that housed the county seat (which are typically more urbanized) were excluded. A total of 36 townships were enrolled from 159 townships. Third, 7 or 8 villages (depending on how many villages had populations more than 800) were selected from each sampled township. If there were no villages with populations of 800 or more, 2 small neighboring villages were combined and considered as 1 village-level sampling unit. A total of 283 villages were enrolled from 903 villages. Finally, our team obtained a list of all registered births over the past 24 months from the local officials in each village, and all caregivers with an infant in the target age range (6-24 months) were enrolled in each sample village. A total of 1,515 caregivers were eligible. In total, 139 eligible caregivers failed to enroll in the study for various reasons, which included that their household had migrated to another city or province for work; they were visiting relatives during the time of the survey; their infant was sick, and the caregiver was not able to be interviewed; or they refused the interview. Of the 1,376 caregivers who enrolled in this study, 1,338 caregivers completed all aspects of the questionnaire, for a response rate of $97.2 \%$. After imputing 20 missing values, the final analytical sample included 1,358 participants.

This study received ethical approval from the Sichuan University Medical Ethical Review Board (approval no. K2018103). Before conducting interviews, trained enumerators introduced the study aims, the process, the potential risks and benefits, the privacy measures that were taken, the rights and duties of the individual, and the contact for the study. Enumerators also presented each household with a standardized document that outlined the same information.

\section{Data Collection}

Data were collected through survey interviews by trained enumerators using a structured survey questionnaire. 


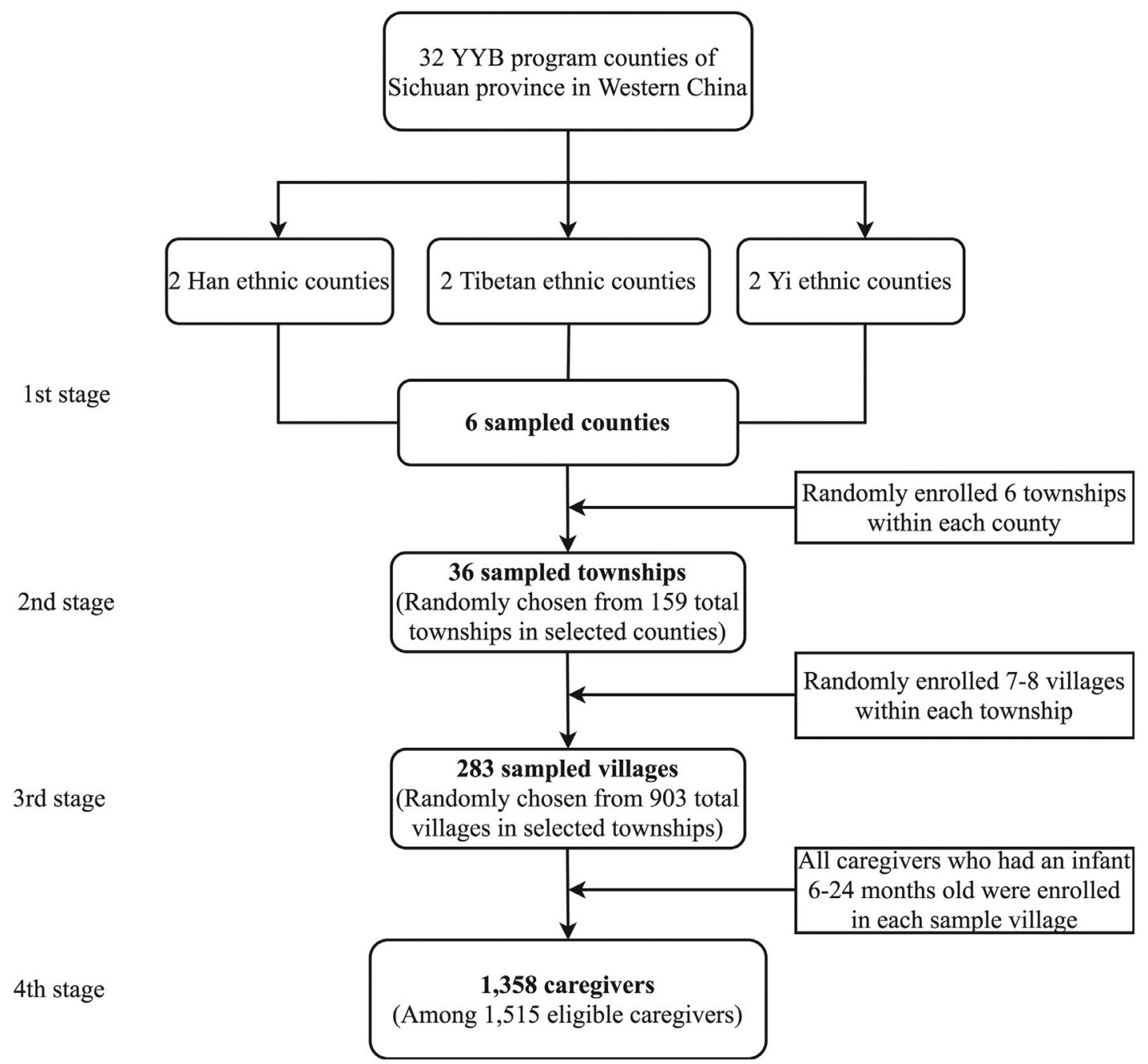

Figure. Flow diagram of the sampling procedure. YYB indicates Ying Yang Bao (a micronutrient powder program conducted in China).

This questionnaire was developed using a comprehensive literature review and 2 rounds of Delphi expert consultation. After assessing the questionnaire and revising it for validity, our enumerators pretested the questionnaire in nonsample villages (details regarding the development of the questionnaire are listed in Supplementary Data).

In Tibetan and Yi communities, local doctors often use local dialects while providing caregivers with information about YYB. For our research team to overcome language barriers presented in minority communities, we used locally trained volunteers to help translate between Mandarin and the local dialect during the survey interviews. The survey collected 3 blocks of data, including YYB adherence, YYB health communication, and demographic characteristics.

The first survey block concerned the outcome of interest in this study, which was caregiver adherence to the YYB program. To assess adherence, each caregiver was asked to report how many packets of YYB they fed their child each week, on average. The WHO and Chinese YYB program offices recommend that children be fed 4-7 packets of YYB per week. $^{4,18,26}$ Nonadherence to YYB was thus defined as feeding children fewer than 4 YYB packets per week.

The second survey block focused on how YYB health information was communicated to caregivers. The nature by which the respondent household had heard about the YYB program was measured in 2 dimensions: communication channels and messages about YYB. To assess communication channels for YYB information, enumerators asked caregivers to select the typical channels through which they received information about YYB, including family members, relatives and friends, village cadres (local officials), village 
doctors, township doctors, and mass media (eg, leaflets, radio, television). To assess the messages about YYB that caregivers received through these channels, our team asked caregivers a series of questions: (1) whether they received any YYB information, (2) whether they were informed about the health benefits of YYB, (3) whether they had been told that YYB was free, and (4) whether they had received information about YYB feeding methods.

The third block concerned the demographic characteristics of caregivers. In this part of the survey, enumerators asked about the caregiver's age, gender, ethnicity, educational level, occupation, and household assets. Ethnicity was self-reported by the caregivers from a list that included Han, Tibetan, and Yi. To assess caregiver educational level, enumerators asked caregivers to choose 1 of 6 response options: no formal education, did not finish primary school, primary school, junior school, high school, and undergraduate or higher level. Because nearly half of the participants in Tibetan and Yi communities had not attended any school, we transformed the responses into a binary variable, in which $0=$ no formal education and $1=$ any amount of formal education. To produce a measure of household wealth, enumerators asked caregivers a series of questions that collected information about the value of each household's assets. A household asset index was calculated using polychoric principal components analysis ${ }^{27,28}$ on the basis of whether the household owned or had access to a water heater, washing machine, refrigerator, air conditioner, television, computer, motorcycle, and car or truck. Using the index, households were grouped into lower levels of assets (asset index score below the mean) and higher levels of assets (asset index score above the mean).

\section{Statistical Analysis}

The empirical strategy included 3 parts. First, descriptive analyses were used to assess the statistical values of the variables that measured the outcomes of interest and the independent variables. Continuous variables were expressed as mean and $\mathrm{SD}$, and categorical variables as numbers and percentages. The missing values were imputed using regression imputation, ${ }^{29,30}$ using the mi impute regress command in Stata for the imputation. $^{29,30}$ Second, we used Chi-square tests of independence to assess differences in adherence to YYB among Han, Tibetan, and Yi ethnic groups and differences in YYB health communication patterns. Finally, to understand how health communication patterns are associated with YYB adherence among Han, Tibetan, and Yi caregivers, we estimated 3 robust logistic regression models for each ethnic group separately. In each regression, the health communication patterns (communication channel and communication message) were predictors, and adherence to YYB was the outcome variable. Each regression model used an adjusted model that controlled for covariates (age, gender, educational level, occupation, and household fixed assets level) to calculate the odds ratio (OR) and the associated 95\% confidence interval (CI). In all cases, we used a cluster-corrected estimator to adjust standard errors for clustering at the township level; $P<0.05$ were considered statistically significant. All statistical analyses were conducted using Stata software (version 14.1, StataCorp, College Station, TX, 2015).

\section{RESULTS}

\section{Demographics of the Caregivers by Ethnicity}

The demographic characteristics of study participants are reported in Table 1. Among the 1,358 caregivers, the average age was 36 years (SD, 13 years). Most caregivers were female $(91.8 \%)$. More than half of the caregivers had a formal education $(54.2 \%)$, and about half were stay-athome parents $(48.2 \%)$. Less than half reported a level of household assets above the mean of the household fixed assets index score (47.3\%). Demographically, Han, Tibetan, and Yi caregivers differed significantly in educational level, occupation, and household asset value $(P<0.05)$.
Adherence to YYB and Health

\section{Communication}

The rates of adherence to YYB are shown in Table 2 . The results show that 1,128 of 1,358 caregivers $(83.1 \%)$ had heard about the YYB program. Significantly more Han caregivers $(94.3 \%)$ knew about YYB than did Tibetan (79.3\%) and Yi (76.3\%) caregivers (both $P<0.05$ ). Among caregivers who had heard about YYB, 52.6\% administered YYB to their child as recommended (4-7 packets per week). Adherence to YYB recommendations varied significantly among the 3 ethnic groups $(P<0.05)$. Yi caregivers reported significantly lower rates of adherence $(47.2 \%)$ than did Han $(55.5 \%)$ and Tibetan $(55.0 \%)$ caregivers $(P<0.05)$.

Table 2 presents the communication channels through which caregivers most frequently received YYB information. Among the 1,128 caregivers who had heard about YYB, the most common communication channel was township doctors (81.4\%), followed by relatives or friends (19.1\%). Among the 3 ethnic groups in the sample, the results showed significant differences in communication channels. Significantly higher percentages of Han caregivers received information from relatives or friends $(30.5 \%)$, mass media (22.8\%), and family members (18.6\%) than did Tibetan and Yi caregivers (all $P<0.05)$. In addition, significantly more Han (87.7\%) and Tibetan (90.2\%) caregivers received YYB information from township doctors as compared with Yi (70.3\%) caregivers (both $P<$ 0.05). In comparison, significantly higher percentages of $\mathrm{Yi}$ caregivers received information from village doctors $(12.5 \%)$ and village cadres $(7.7 \%)$ as compared with Han and Tibetan caregivers (both $P<0.05$ ).

Table 2 also provides information about the messages about YYB that caregivers received. Caregivers primarily reported receiving messages on the health benefits of YYB (45.4\%), followed by the message that YYB is free $(28.0 \%)$. These percentages are consistent among Han and Tibetan caregivers who received YYB information. However, among Yi caregivers, approximately one third knew no more than the YYB name and had not received any other information about 
Table 1. Demographics of Caregivers by Ethnicity in Rural Western China $(n=1,358)$

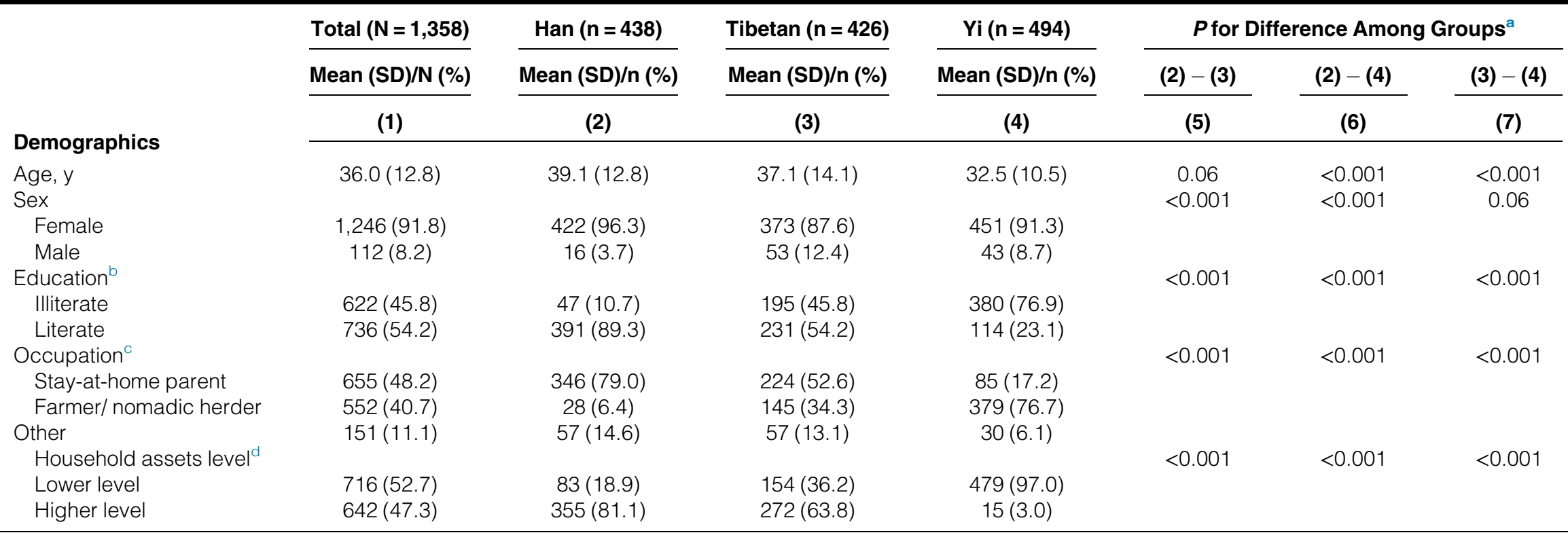

SD indicates standard deviation.

${ }^{a}$ Analysis of variance s were conducted with the oneway command and bonferroni option in Stata. Using these statistical tools, the group differences of the sample's continuous variables, such as caregiver age, were analyzed. To examine group differences between categorical variables, such as caregiver sex, education, occupation, and household assets, chi-square tests of independence between Han and Tibetan ethnic groups, Han and Yi ethnic groups, and Tibetan and Yi ethnic groups were conducted, for which $P<0.05$ were considered statistically significant; ${ }^{\mathrm{b}}$ To assess caregiver educational level, caregivers were asked to choose 1 of 6 response options: no formal education, did not finish primary school, primary school, junior school, high school, and undergraduate or higher level. We transformed the responses into a binary variable, in which $0=$ no formal education and $1=$ any amount of formal education; ${ }^{\circ}$ The occupation stay-at-home parent refers to caregivers responsible for staying home and caring for the child. The occupation farmer/nomadic herder was found predominantly in Tibetan communities (27.7\%), and there are no nomadic herders among Han and Yi caregivers. Other occupations include off-farm part-time jobs and self-employment; ${ }^{\mathrm{d} A} \mathrm{~A}$ household fixed assets score was developed using polychoric principal component analysis on the basis of whether the household owned or had access to a water heater, washing machine, refrigerator, air conditioner, television, computer, motorcycles, and car or truck. Households were then divided into 2 groups: lower levels of assets (asset index score below the mean) and higher levels of assets (asset index score above the mean). 
Table 2. Feeding Practice and Health Communication on YYB Program of Caregivers by Ethnicity in Rural Western China $(n=1,358)$

\begin{tabular}{|c|c|c|c|c|c|c|c|}
\hline & \multirow{2}{*}{$\frac{\text { Total }(\mathrm{N}=1,358)}{\mathrm{N}(\%)}$} & \multirow{2}{*}{$\frac{\operatorname{Han}(n=438)}{n(\%)}$} & \multirow{2}{*}{$\frac{\text { Tibetan }(n=426)}{n(\%)}$} & \multirow{2}{*}{$\frac{Y i(n=494)}{n(\%)}$} & \multicolumn{3}{|c|}{$P$ for Difference Among Groups ${ }^{a}$} \\
\hline & & & & & (2) - (3) & (2) - (4) & (3) - (4) \\
\hline & (1) & (2) & (3) & (4) & (5) & (6) & (7) \\
\hline \multicolumn{8}{|l|}{ YYB Health Communication } \\
\hline Heard about YYB program $(N=1,358)^{b}$ & & & & & $<0.001$ & $<0.001$ & 0.27 \\
\hline Yes & $1,128(83.1)$ & $413(94.3)$ & $338(79.3)$ & $377(76.3)$ & & & \\
\hline No & $230(16.9)$ & $25(5.7)$ & $88(20.7)$ & $117(23.7)$ & & & \\
\hline $\begin{array}{l}\text { Average no. of YYB packets fed to child/wk } \\
(n=1,128)^{\mathrm{C}}\end{array}$ & & & & & 0.46 & 0.02 & 0.04 \\
\hline$<4$ & $535(47.4)$ & $184(44.5)$ & $152(45.0)$ & $199(52.8)$ & & & \\
\hline $4-7^{d}$ & $593(52.6)$ & $229(55.5)$ & $186(55.0)$ & $178(47.2)$ & & & \\
\hline \multicolumn{8}{|l|}{ YYB communication channels $(n=1,128)^{C}$} \\
\hline Township doctors & & & & & 0.26 & $<0.001$ & $<0.001$ \\
\hline Yes & $952(81.4)$ & $362(87.7)$ & $305(90.2)$ & $265(70.3)$ & & & \\
\hline No & $218(18.6)$ & $51(12.3)$ & $33(9.8)$ & $112(29.7)$ & & & \\
\hline Relatives or friends & & & & & $<0.001$ & $<0.001$ & 0.73 \\
\hline Yes & $216(19.1)$ & $126(30.5)$ & $41(12.1)$ & $49(13.0)$ & & & \\
\hline No & $912(80.9)$ & $287(69.5)$ & $297(87.9)$ & $328(87.0)$ & & & \\
\hline Mass media $^{e}$ & & & & & $<0.001$ & $<0.001$ & 0.79 \\
\hline Yes & $130(11.1)$ & $94(22.8)$ & $13(3.8)$ & $16(4.2)$ & & & \\
\hline No & $1,040(88.9)$ & $319(77.2)$ & $325(96.2)$ & $361(95.8)$ & & & \\
\hline Family members & & & & & $<0.001$ & $<0.001$ & 0.12 \\
\hline Yes & $116(9.9)$ & $77(18.6)$ & $20(5.9)$ & $13(3.4)$ & & & \\
\hline No & $1,054(90.1)$ & $336(81.4)$ & $318(94.1)$ & $364(96.6)$ & & & \\
\hline Village doctors & & & & & 0.20 & $<0.001$ & $<0.001$ \\
\hline Yes & $51(4.4)$ & $2(0.5)$ & $0(0.0)$ & $47(12.5)$ & & & \\
\hline No & $1,119(95.6)$ & $411(99.5)$ & $338(100.0)$ & $330(87.5)$ & & & \\
\hline Village cadres & & & & & 0.12 & $<0.001$ & $<0.001$ \\
\hline Yes & $36(3.1)$ & $1(0.2)$ & $4(1.2)$ & $29(7.7)$ & & & \\
\hline No & $1,134(96.9)$ & $412(99.8)$ & $334(98.8)$ & 348 (92.3) & & & \\
\hline Messages about YYB $(n=1,128)^{c}$ & & & & & 0.001 & $<0.001$ & $<0.001$ \\
\hline YYB benefits ${ }^{\dagger}$ & $512(45.4)$ & $185(49.7)$ & $184(54.4)$ & $123(32.6)$ & & & \\
\hline YYB is free & $316(28.0)$ & $152(36.8)$ & $83(24.6)$ & $81(21.5)$ & & & \\
\hline Did not receive any YYB information ${ }^{\dagger}$ & $210(18.6)$ & $34(8.2)$ & $49(14.5)$ & $127(33.7)$ & & & \\
\hline Methods of feeding $\mathrm{YYB}^{\dagger}$ & $90(8.0)$ & $22(5.3)$ & $22(6.5)$ & $46(12.2)$ & & & \\
\hline
\end{tabular}

YYB indicates Ying Yang Bao (a micronutrient powder program conducted in China).

${ }^{a}$ Analysis of variances were conducted with the oneway command and Bonferroni option in Stata. Using these statistical tools, the group differences of the sample's continuous variables, such as caregiver age, were analyzed. To examine group differences between categorical variables, such as caregiver sex, education, occupation, and household assets, chi-square tests of independence between Han and Tibetan ethnic groups, Han and Yi ethnic groups, and Tibetan and Yi ethnic groups were conducted, for which $P<0.05$ were considered statistically significant; ${ }^{b}$ Caregivers $(N=1,358)$; ${ }^{\circ}$ Caregivers who heard about the YYB program ( $\left.n=1,128\right)$; ${ }^{d} Y Y B$ adherence was defined as feeding infants 4 to 7 YYB packets per week; ${ }^{e}$ Mass media, including leaflets, radios, and televisions; ${ }^{f} Y Y B$ benefits means "I heard that YYB gives health benefits to my child (such as preventing malnutrition and increasing immunity)", did not receive any YYB information means "I just know the YYB name and did not receive any other YYB information", and methods of feeding YYB means "I heard that I need to use warm water to stir YYB and then to mix YYB with the child's favorite food." 
YYB (33.7\%). Similarly, few Yi caregivers knew about the health benefits of YYB (32.6\%), and even fewer knew that YYB was free (21.5\%). Among all 3 groups, information on methods of feeding YYB reached only a small percentage of caregivers (Han, 5.3\%; Tibetan, 6.5\%; and Yi, 12.2\%).

The Association Between Health Communication and Adherence to YYB

Table 3 shows the correlations between health communication (communication channels and messages about YYB) and YYB adherence among caregivers, controlling for demographic variables. Among all 3 ethnic groups, receiving information from township doctors was correlated with increased adherence to YYB (Han: odds ratio [OR], 2.18; 95\% CI, 1.11 -4.29; Tibetan: OR, 7.22; 95\% CI, 1.97 -26.55; Yi: OR, 2.99; 95\% CI, 1.55 -5.75). However, apart from township doctors, each ethnic group showed unique correlations between YYB adherence and a specific communication channel. Receiving information via mass media was significantly associated with YYB adherence among
Han caregivers (OR, 1.87; 95\% CI, 1.05 -3.31). Among Tibetan caregivers, communication with family members was significantly linked to adherence to YYB (OR, 4.86; 95\% CI, 1.45 -16.29), and village doctors were significantly associated with Yi adherence to YYB (OR, 6.63; 95\% CI, 3.46 $-12.73)$.

Messages about YYB also showed varying associations to YYB adherence among the 3 ethnic groups. Tibetan caregivers were more likely to adhere to YYB if they obtained information on the health benefits of YYB (OR, 2.00; 95\% CI, 1.03-3.90). For Yi caregivers, receiving information on the health benefits of YYB (OR, 2.30; 95\% CI, 1.14-4.62) and information about feeding methods (OR, 2.48; 95\% CI, 1.22-5.03) were associated with YYB adherence. In contrast, no types of YYB information were significantly associated with YYB adherence among Han caregivers.

\section{DISCUSSION}

This study examined the associations between various health communication patterns and YYB adherence among ethnic Han, Tibetan, and Yi caregivers in rural western China. The results found that rates of adherence to YYB varied significantly among the 3 ethnic groups. Surprisingly, all 3 ethnic groups received YYB information primarily from township doctors, which was shown to be positively associated with adherence rates. Apart from township doctors, this study found significant differences between health communication patterns and adherence to YYB among Han, Tibetan, and Yi caregivers.

Although YYB packets are safe and effective, in practice, the effectiveness of YYB depends on community adherence. $^{31}$ Across the 3 ethnic groups in this study, just more than half of the caregivers adhered to YYB feeding instructions, far below the national average of $70 \% .{ }^{17}$ Although the difference in adherence between Han and Tibetan caregivers was not significant, the difference between Han and Yi caregivers, as well as between Tibetan and Yi caregivers, was significant. Previous studies of YYB have found that the variance in caregivers' adherence between ethnic groups may be explained by differences in SES (including household assets and education

Table 3. Association Between Health Communication and Adherence to YYB Among Children's Caregivers from Different Ethnicity in Rural Western China $(n=1,128)$

\begin{tabular}{|c|c|c|c|c|c|c|}
\hline & & & Adherer & to YYB & & \\
\hline & Han $(n$ & 13) & Tibetan & $=338)$ & Yi (n & 77) \\
\hline & Adjusted OR & $95 \% \mathrm{Cl}$ & Adjusted OR & $95 \% \mathrm{Cl}$ & Adjusted OR & $95 \% \mathrm{Cl}$ \\
\hline & (1) & (2) & (3) & (4) & (5) & (6) \\
\hline YYB Health Communication & & & & & & \\
\hline Communication channel of YYB informa & & & & & & \\
\hline Township doctors & $2.18^{\star \star}$ & $1.11-4.29$ & $7.22^{\star \star}$ & $1.97-26.55$ & $2.99^{* * *}$ & $1.55-5.75$ \\
\hline Mass media & $1.87^{*}$ & $1.05-3.31$ & 1.05 & $0.25-4.34$ & 0.62 & $0.24-1.61$ \\
\hline Family members & 1.00 & $0.53-1.90$ & $4.86^{\star}$ & $1.45-16.29$ & 1.37 & $0.37-5.08$ \\
\hline Village doctors & - & - & - & - & $6.63^{* * *}$ & $3.46-12.73$ \\
\hline Village cadres & - & - & 1.60 & $0.18-14.12$ & 1.10 & $0.42-2.87$ \\
\hline Relatives or friends & 1.03 & $0.72-1.47$ & 1.06 & $0.36-3.13$ & 1.38 & $0.73-2.61$ \\
\hline Main communication messages of YYB & & & & & & \\
\hline Did not receive any YYB information & Reference & & Reference & & Reference & \\
\hline YYB benefits & 1.13 & $0.53-2.43$ & $2.00^{*}$ & $1.03-3.90$ & $2.30^{\star *}$ & $1.14-4.62$ \\
\hline Methods of feeding YYB & 1.40 & $0.49-4.02$ & 1.28 & $0.47-3.50$ & $2.48^{*}$ & $1.22-5.03$ \\
\hline YYB is free & 1.00 & $0.48-2.09$ & 0.83 & $0.32-2.17$ & 1.13 & $0.60-2.11$ \\
\hline
\end{tabular}

$\mathrm{Cl}$ indicates confidence interval; OR, odds ratio; YYB, Ying Yang Bao, (a micronutrient powder program conducted in China). ${ }^{\star} P<0.05 ;{ }^{* *} P<0.01 ;{ }^{* *} P<0.001$.

Note: Adherence to YYB was defined as feeding children 4 to 7 YYB sachets/wk by caregivers. Clustering is at the township level. For each regression, we fit the model after controlling for caregiver demographic variables, including age, sex, educational level, occupation, and household fixed assets level. All variables in the dimension of the communication channel of YYB information are dummy variables. 


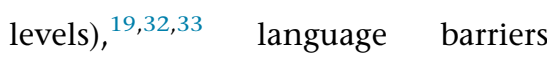
(Mandarin is the primary language used in mass media), ${ }^{19,34}$ and differences in communication channels and messages in regard to YYB information in Han and minority areas. ${ }^{35}$

Among Han, Tibetan, and Yi groups, the findings indicated that township doctors played an important role in promoting YYB adherence. Township doctors were the most frequently reported communication channel for YYB information. Township doctors were also the only significant determinant of caregiver adherence to YYB common across all 3 ethnic groups. Additional analysis revealed that more than two-thirds of the overall sample received YYB information from township doctors, typically when they brought their children to the township health center for vaccinations or physical examinations. This is a far higher percentage than other YYB counties in China, in which $<20 \%$ of caregivers reported receiving YYB packets from township doctors. ${ }^{36}$

However, aside from township doctors, each ethnic group differed in terms of the most common communication channels through which caregivers received messages associated with YYB adherence. Among Han caregivers, communicating YYB information via mass media (such as leaflets, radio, or television) effectively promoted YYB adherence, which is consistent with previous studies in Han communities of China. ${ }^{37,38}$ This association can be explained by the relatively high educational and household fixed assets levels found among Han caregivers, both in this study sample and nationally. ${ }^{16}$ Greater access to mass media (because of higher levels of household assets) may provide increased exposure to information in the case of Han caregivers. ${ }^{39,40}$ Another possible reason that mass media is most accessible to Han communities could be their higher Mandarin literacy rates. Given that most mass media is in Mandarin (which is the native language for many Han households), it seems likely that language plays an essential role in promoting YYB adherence in Han communities. Unlike Han caregivers, the communication methods predominantly used by minorities are likely influenced by local cultural norms, which contribute to the YYB adherence in minority communities. $^{41,42}$

An example of such cultural norms was found in the sample of Tibetan caregivers. The results indicated that receiving health information from family members was most likely to increase YYB adherence in Tibetan communities. This may be influenced by the nomadic lifestyle of many Tibetan caregivers (27.7\%) in the sample, who spend most of the year herding animals with other family members (and with little access to other channels of communication). In addition, the majority of the sampled caregivers who reported being stay-at-home parents $(52.6 \%)$ participate in the nomadic lifestyle but are tasked with raising children rather than taking an active role in herding animals. ${ }^{43,44}$ These families are typically isolated during nomadic periods (April to November each year). ${ }^{42,43}$ This isolation means that caregivers communicate almost exclusively with family members during nomadic periods. As such, interpersonal community-based communication strategies that educate family units may be most effective in promoting YYB adherence among Tibetan caregivers.

Similarly, the results show that interpersonal relationships are also important to Yi caregiver adherence to YYB, as Yi caregiver adherence is predominantly influenced by village doctors. In many Yi communities, village doctors are more accessible to Yi caregivers because of their connection to the community (they are usually members of the village), including proximity to Yi households and mastery of the local dialect. ${ }^{45}$ Thus, although it is clear that township doctors play a key role in improving Yi caregiver adherence, village doctors may be even more effective in communicating YYB information because of their accessibility to and familiarity with the individuals in $\mathrm{Yi}$ communities. The findings indicate that, in the case of Yi communities, further utilization of village doctors may increase YYB adherence.

In addition to differences in the effectiveness of different communication channels, the nature of the messages received about YYB also differed among ethnic groups (as well as having different impacts on adherence). Although no messages about YYB were significant for Han caregivers, this study found that adherence rates among the Tibetan and Yi communities increased significantly when caregivers were given information about the health benefits of YYB (eg, preventing anemia, strengthening immunity). These findings are consistent with previous research highlighting the importance of caregiver knowledge and increased adherence rates. ${ }^{19}$

Taken together, the findings emphasize the necessity to promote YYB adherence in rural western China, particularly among minority caregivers. The results indicate that interpersonal community-based interventions are most appropriate for minority populations. Specifically, for Tibetan caregivers, future interventions could consider the influence of the family on YYB adherence and provide greater outreach on YYB health benefits to family units. For Yi caregivers, village doctors may be effective in bridging unique cultural and linguistic practices in the $\mathrm{Yi}$ community.

This study examined the association between health communication patterns and YYB adherence among Han, Tibetan, and Yi ethnic groups in western China and provides insight into how culturally appropriate health communication strategies can effectively be used to promote YYB adherence in western China as well as in other ethnically diverse LMICs.

This study is also subject to certain limitations. First, because the data on YYB feeding practices were based on caregiver recall, this study could not rule out the possibility of recall bias. To increase accuracy, future studies might use enumerators to count the number of unused packets per month in addition to asking the caregiver to recall the number of YYB packets fed to infants. Second, caregivers were not asked follow-up questions about the YYB messages they had received from each YYB channel they had affirmed using; rather, they were asked about the messages received from YYB communication channels, 
generally. Future study is needed to capture more detailed information about the type of information received through each YYB channel. Third, for a more in-depth understanding of how cultural norms influence caregiver adherence among diverse ethnic populations, future research may consider using qualitative and/or mixed-method approaches. Fourth, although measures were taken before the interviews to reduce bias during the survey because of the quality of the translations that was needed between respondent and surveyor in minority areas (all measures are listed in Supplementary Data), it is possible that the use of locally trained volunteers to translate the survey may have resulted in the inconsistent presentation of the survey questions.

\section{IMPLICATIONS FOR RESEARCH AND PRACTICE}

The findings of this study underscore the importance of designing culturally tailored health communication strategies to improve adherence to micronutrient intervention programs among caregivers with diverse ethnic backgrounds. The findings indicate that a culturally specific, interpersonal health communication strategy can significantly increase YYB adherence among ethnically diverse rural caregivers. Given that township doctors play a critical role in caregiver adherence to YYB feeding instructions across Han, Tibetan, and Yi groups, training township doctors to communicate YYB information more effectively may broadly increase YYB adherence. This study indicated that minority communities display the lowest rates of adherence, calling for community-based interventions to be implemented to promote better YYB practices in minority populations in rural China.

\section{ACKNOWLEDGMENTS}

This research was funded by the National Natural Science Foundation of China, grant no. 71874114. The authors would like to thank the collaboration of the local officials and all participants from each of the participating sites in this research for providing substantial assistance in collecting our survey data.

\section{SUPPLEMENTARY DATA}

Supplementary data related to this article can be found at https://doi. org/10.1016/j.jneb.2021.07.014.

\section{REFERENCES}

1. United Nations Children's Fund, World Health Organization, International Bank for Reconstruction and Development. Levels and trends in child malnutrition: key findings of the 2020 edition of the joint child malnutrition estimates. World Health Organization; 2020. https://www.who.int/ publications-detail/jme-2020-edition. Accessed May 22, 2021.

2. Bailey RL, West KP, Black RE. The epidemiology of global micronutrient deficiencies. Ann Nutr Metab. 2015;66 (Suppl 2):22-33. (2).

3. Sally GM, Cornelius A. A review of studies on the effect of iron deficiency on cognitive development in children. $J$ Nutr. 2001;131(2S-2):649S-666S. discussion 666S-668S.

4. World Health Organization. Use of Multiple Micronutrient Powders for Home Fortification of Foods Consumed by Infants and Children 6-23 Months of Age. WHO; 2011. https://apps.who.int/iris/bitstream/ handle/10665/44651/9789241502047_ eng.pdf; jsessionid=88FAC3F98AAE7BE8DD 2979E2590B 0FCE? sequence=1. Accessed May 22, 2021.

5. Zhou L, Li CY, Jin JZ, Liu Q, Jin GH. Meta-analysis of the intervention effect of complementary food supplement on infants and young children in developing countries. Mod Prev Med. 2018;45. $3694-3698+3726$.

6. Roschnik N, Diarra H, Dicko Y, et al. Adherence and acceptability of community and a distribution of micronutrient powders in Southern Mali. Matern Child Nutr. 2019;15(Suppl 5):e12831.

7. Stephen K, Hyun RJ, Klaus K, Saskia DP, Joel G. Understanding low usage of micronutrient powder in the Kakuma refugee camp, Kenya: findings from a qualitative study. Food Nutr Bull. 2011;32:292-303.

8. Haribondhu S, Fakhar UM, Catherine $\mathrm{H}$, Tahmeed A. Factors influencing child feeding practices related to home fortification with micronutrient powder among caregivers of under- 5 children in Bangladesh. Food Nutr Bull. 2016;37:340-352.

9. Tumilowicz A, Schnefke CH, Neufeld LM, Pelto GH. Toward a better understanding of adherence to micronutrient powders: generating theories to guide program design and evaluation based on a review of published results. Curr Dev Nutr. 2017;1:e001123.

10. Michaux K, Anema A, Green T, Smith L, McLean J. Home fortification with micronutrient powders: lessons learned from formative research across six countries. Sight and Life. 2014;28:26-35.

11. Hilary CK, Rosario B, Melissa A, Varinia A. Promoting multiting Lifer und powders (MNP) in Peru: acceptance by caregivers and role of health personnel. Matern Child Nutr. 2016;12:152-163.

12. Loechl CU, Menon P, Arimond M, et al. Using programme theory to assess the feasibility of delivering micronutrient sprinkles through a food-assisted maternal and child health and nutrition programme in rural Haiti. Matern Child Nutr. 2009;5:33-48.

13. Nie JC, Yang J, Zhang LF, Li Y, Yang J. 中国农村贫困地区贞幼儿贫血现状 及影响因素分析 [Current status and influencing factors of infant anemia in poor rural areas of Western China]. J East China Norm Univ (Educ Sci). 2019;37:58-69.

14. Sun C, Meng S, Wang QZ, et al. Effect of utilization of child health services on the nutrition and growth status of infants in poor rural areas in Sichuan and Gansu province. $J$ Hyg Res. 2019;48:745-750

15. National Health Commission. All-China Women's Federation. Chidren's nutrition improvement project program in poor areas. http://www.gov.cn/gzdt/ 2013-11/29/content_2538706.htm. Accessed May 22, 2021.

16. National Bureau of Statistics of China. China statistical yearbook. http://www. stats.gov.cn/tjsj/ndsj/2019/indexch.htm. Accessed May 22, 2021.

17. National Health Commission. The Ministry of Finance, National Administration of Traditional Chinese Medicine. Notice on preparing to the basic public health service in 2019. http:// www.gov.cn/xinwen/2019-09/05/ content_5427467.htm. Accessed May 22, 2021.

18. National Health Commission. AllChina Womenwomen's Federation. 
The technical protocol of chidren nutrition improvement program in poor areas. http://www.doc88.com/ p-3893134660583.html. Accessed May 22, 2021

19. Li FY, Fang X, Liu XD, Wang M, Yang L. Adherence to YYB program and its influence factors among caregivers in poor rural Gansu province. Matern Child Health Care China. 2019;34: 4851-4855.

20. Zhou H, Sun S, Luo RF, et al. Impact of text message reminders on caregivers' adherence to a home fortification program against child anemia in rural Western China: a cluster-randomized controlled trial. Am J Public Health. 2016;106:1256-1262.

21. Wu Q, Zhang YF, Chang SY, et al. Monitoring and evaluating the adherence to a complementary food supplement (Ying Yang Bao) among young children in rural Qinghai, China: a mixed methods evaluation study. J Glob Health. 2017;7:011101.

22. Wang QZ, Wu YJ, Ye RX, et al. Factors analysis on caregivers' compliance behaviors in nutrition packages feeding by PRECEDE theory in poor rural areas of Southern Shaanxi Province. J Hyg Res. 2018;47:599-604.

23. Sichuan Bureau of Statistics. Sichuan Statistical Yearbook-2019. http://tjj.sc. gov.cn/tjnj/cs/2019/zk/indexch.htm. Accessed May 22, 2021.

24. Li H. 现代视角下的西部多民族文化 探索 [Exploration of multi-ethnic culture in the Western China from the modern perspective]. J Humanit. 2006; 4:86-90.

25. Qu PF, Yan H, Zhang Y, et al. Investigation on ethnic differences of supplementary feeding for infants in rural areas of Western China. J Zhejiang Univ Sci B (Biomed Biotechnol). 2018;19:71-78.

26. Chinese Center for Disease Control and Prevention. Notice on printing and distributing The Program of Children nutrition improvement project in poor areas in 2014. http://www.chinanutri.cn/gzrw_132/pkdqetyygssdxm/ 201412/t20141229_108522.html. Accessed May 22, 2021.

27. Amouzou A. Socio-economic differences in health, nutrition, and population in Chad. Urban Geogr. 2007;32:568588.

28. Kolenikov S, Angeles G. Socioeconomic status measurement with discrete proxy variables: is principal component analysis a reliable answer? Rev Income Wealth. 2010;55:128-165.

29. Royston P. Multiple imputation of missing values: further update of ice, with an emphasis on categorical variables. STATA J. 2009;9:466-477.

30. Lee KJ, Carlin JB. Multiple imputation for missing data: fully conditional specification versus multivariate normal imputation. Am J Epidemiol. 2010; 171:624-632.

31. Xu J, Li Y, Huo JS, Sun J, Huang J. Supplementing fortified soybean powder reduced anemia in infants and young children aged 6-24 months. Nutr Res. 2019;3:21-33.

32. Zhou X, Fang JQ, Luo JY, et al. Factors associated with taking Yingyangbao efficiently among infants and young children aged 6-24 months in poor rural areas of Hunan province, China. $J$ Hyg Res. 2017;46:256-261.

33. Zhang WJ, Yang HX, Li PX, Peng MQ. 某县贫困地区儿童营养改善项 目营养包服用影响因素分析 [Analysis on the influence factors about taking nutrition package of "projects on children nutrition improvement in poor areas in some country]. Chin J Women Child Health. 2015;6:26-29.

34. Shan GH. 甘肃民族地区信息传播的 现状及对策研究 [Research on the communication status and improve measures in Gansu minority areas]. Eur Educ Research. 2012;33:62-66.

35. Qu PF, Ya Z, Li JM, et al. Complementary feeding patterns among ethnic groups in rural Western China. J Zhejiang Univ Sci B. 2018;19:71-78.

36. China Development Research Foundation. Evaluation report of children's nutrition improvement project in poor rural areas in China; 2016. https://cdrf. org.cn/jjh/pdf/pinkun.pdf. Accessed May 22, 2021.

37. Ding J, Liu YJ, Liu YY, Wu YJ, Gao F, Gao S. Effect of an intervention study using mobile phone services and internet to promote breastfeeding during infant period. Chin Nurs Manag. 2016;16:527-530.

38. Ye AL, Lin LN, Zeng SP. Influence of health education intervention on behavior of community breastfed and growth and development of infant. China Med Pharm. 2017;7: 254-256.

39. Kong ZM. Review of research on mass communication and rural social development. Youth Journalist. 2008;17:3031.

40. Zhou Y, Liu XY. A research on rural audience uses and gratifications of mass media, based on the fieldwork in Wanshui village in eastern Sichuan. J Int Commun. 2011;33:72-78.

41. Liu TT, Li YH, Li YB, Jin XZ, Wang XL。四川省凉山彝族自治州农村地 区艾滋病健康教育现状调查及策略 建议 [Investigation and suggestions to AIDS health education in rural areas of Liangshan Yi Autonomous prefecture]. Chin J Health Educ. 2010;26:334-337.

42. Wang X, Shi SL, Zhang DG. Rangeland nomadic culture of the Tibetan (II) - the culture of rangeland and production. Grassland and Turf. 2011;31:1-4.

43. Ruoergai Government. The nomadic culture. http://www.ruoergai.gov.cn/ regxrmzf/c100135/202105/1f5ec028c 48d4f908a77ae5ba6e7c3c9.shtml. Accessed May 22, 2021.

44. Sun JX. 藏区城镇,农业, 牧业社区文 化比较研究 - 以甘南夏河县为例 [Comparative study on community culture among town, farm belt and pasturing area of Tibetan-case study of Xiahe County Gannan]. Nationalities Res Qinghai. 2006;17:33-41.

45. Lei YT, Zhang B, Song Y. Health education information dissemination modes and practicability of health education content of IMCH program and IMCHD program in 5 provinces/autonomous regions in China: a qualitative study. Matern Child Health Care China. 2017;32:37013704.

\section{ORCID}

Huan Zhou: http://orcid.org/00000002-6709-5134 